\title{
EVOLUCIÓN HISTÓRICA SOBRE LAMODELIZACIÓN DEL ESPACIO URBANO EN GEOGRAFÍA
}

Santiago Linares ${ }^{1}$

\section{Resumen}

En Geografía, el concepto de"modelo urbano" hace referencia a la explicitación de funciones y procesos que generan estructuras espaciales urbanas en términos de uso de la tierra, población, empleo y transporte, permitiendo probar determinadas teorías de localización con datos y generar escenarios futuros de distribución, entendiendo por defecto a la modelización, como el proceso de identificar la teoría apropiada, traducir esto en un modelo matemático o formal y luego confrontar el modelo con datos para que pueda ser calibrado, validado y verificado antes de su uso en la predicción. Si bien, desde su implementación inicial los modelos son vistos como dispositivos o mecanismos para poner a prueba la idoneidad de una teoría; en la actualidad, el uso de modelos se ha extendido más allá de este enfoque hipotético-deductivo que le dio origen y responden a la necesidad analítica de comprender y manejar la complejidad que supone el estudio la realidad.En este artículo, se revisany discuten los diferentes enfoques sobre la modelización del espacio urbano en la investigación geográfica, los planteamientos teóricos subyacentes y las debilidades adjudicadas a cada uno.

Palabras claves: Modelización urbana; Sistemas de Información Geográfica; Modelos en Geografía.

\section{EVOLUÇÃO HISTÓRICA SOBRE A MODELAGEM DO ESPAÇO URBANO NA GEOGRAFIA}

\section{Resumo}

Em geografia, o conceito de "modelo urbano" refere-se a explicação das funções e processos que geram estruturas espaciais urbanas em termos de uso da terra, população, emprego e transportes, o que permite testar algumas teorias de dados de localização e gerar cenários futuros distribuição, ouseja, a modelagem como padrão o processo de identificação apropriado teoria, traduzi-lo em um modelo matemático ou formal e, em seguida, confrontar com o modelo de dados, de modo que ele pode ser calibrado, validado e verificado antes da sua utilização na predição. Enquanto, a partir de modelos de implantação inicial são vistos como dispositivos ou mecanismos para testar a adequação de uma teoria; Actualmente, a utilização de modelos se espalhou para além desta abordagem hipotético-dedutivo que deuorigemao e responder à necessidade analítica para compreender e gerenciar a complexidade da realidadeestudo. Neste artigo, vamos analisar e discutir as diferentes

\footnotetext{
${ }^{1}$ Magister en Teledetección y Sistemas de Información Geográfica por la Universidad Nacional del Centro de la Provincia de Buenos Aires (UNCPBA) y doctor en Geografía por la Universidad Nacional del Sur (UNS).Investigador Asistente del Consejo Nacional de Investigaciones Científicas y Técnicas (CONICET) de Argentina. Docente del Departamento de Geografía de la Facultad de Ciencias Humanas (UNCPBA). Email: slinares@fch.unicen.edu.ar
}

Sociedade e Território - Natal. Vol. 28, N. 2, p. 23 - 41. Jun./Dez. de 2016 
abordagens quanto à concepção do espaço urbano na pesquisa geográfica, as abordagens teóricas subjacentes e fracos atribuído a cada um.

Palavras-chaves:Modelagem urbana; Sistemas de Informação Geográfica; Modelos em Geografia.

\title{
HISTORICAL EVOLUTION OF URBAN SPACE MODELING IN GEOGRAPHY
}

\begin{abstract}
In Geography, the concept of "urban model" refers to the explanation of functions and processes generated byurbans patial structures in terms of land use, population, employment and transportation. Distribution, understandingby default modeling, as the process of identify ingtheap propria tetheory, translatethisinto a mathematicalor formal model and then confronting the model with data so thatit can be calibrated, validated and verifiedbeforeIts use in prediction. Although, fromitsinitial implementation the models are seen as devices ormechanisms to test thesuitability of a theory; At present, the use of models has extended beyond this hy pothetical-deductive approach that gaverise to and respond to theanalyticalneed to understand and complete the complexity of the study of reality. In thisarticle, wereview and discuss the different approaches to urbans pace modeling in geographicresearch, theunderlying theoretical approaches and the weaknesses adjudicate edeach.
\end{abstract}

Keywords: Urban modeling; Geographic Information Systems; Models in Geography.

\section{INTRODUCCIÓN}

El uso de modelos en la investigación urbana se remonta al modelo clásico de localización agrícola de von Thünen, publicado en su famoso libro Der IsolierteStaat (1826). En él, este autor considera la relación de tres factores: la distancia de los agricultores al mercado, los precios recibidos por los agricultores por sus productos y la renta de la tierra. Sobre la base de un análisis econométrico de los Estados de Mecklemburgo en el Norte de Alemania, el autor argumenta que la intensidad del uso de la tierra era inversamente proporcional al costo de transporte o a la distancia del mercado. En un «estado aislado» con un solo centro de la ciudad como mercado único y una llanura uniforme que rodea la ciudad, se genera un patrón concéntrico de uso de la tierra con el menor uso intensivo de ésta situada lo más lejos del centro de la ciudad.

Los posteriores modelos sobre el desarrollo urbano se encuentran muy relacionados con el modelo de von Thünen mencionado. Pero específicamente orientados a la estructura 
urbana interna, podemos afirmar que, sin duda, existe una clara inflexión a partir de los modelos clásicos sobre el crecimiento y patrones de uso del suelo de Burgess (1925), quién publica el modelo de los anillos concéntricos; posteriormente Hoyt (1939) que plantea el modelo de los sectores y, Harris y Ullman (1945) quienes formulan el modelo de los núcleos múltiples. Estos modelos tienen como factor común que se basaron en la comprensión del desarrollo urbano desde el distrito central de negocios (CDB) hacia el exterior.

No obstante, el uso generalizado de modelos en Geografía urbana se produjo durante el período de la denominada «revolución cuantitativa», que se inició a finales de 1950 y se extendió hasta finales de 1960 (BATTY, 1981). Este desarrollo proviene casi exclusivamente desde los Estados Unidos, como resultado de necesidades prácticas coyunturales y del surgimiento de novedosas aplicaciones informáticas. Entre las necesidades prácticas existió un aumentó descomunal de automóviles en circulación durante la década de 1940 y principios de 1950, que llevó a que aquellas ciudades encorsetadas en sus estructuras físicas tradicionales, no soportaran la «nueva movilidad» de sus habitantes. Así, se sentaron las bases para el desarrollo de varios modelos de transporte que fueron consolidándose hacia finales de 1950. Posteriormente, el desarrollo de la informática profundizó esta tendencia al proporcionar un entorno digital para trabajar con modelos matemáticos complejos. Durante este período, una gran variedad de tipos de modelos urbanos se desarrollaron, siendo los temas de aplicación más frecuentes el uso del suelo, el transporte, la población y actividades económicas urbanas. Estos modelos fueron considerados por los planificadores como laboratorios artificiales para experimentar con estructuras urbanas reales.

El énfasis de estos modelos estuvo tradicionalmente orientado a la «sofisticación técnica» del modelado y no a sus «fundamentos teóricos», razón por la cual fueron seriamente criticados a finales de 1970 llevando a un cambio radical sobre el abordaje del desarrollo urbano y pasando de un uso intensivo de modelos matemáticos a análisis fundamentalmente cualitativos. Esta tradición se mantuvo hasta fines de 1980, cuando los enfoques desde la complejidad van a proporcionar alternativas prácticas de entender y estudiar a las ciudades como sistemas evolutivos y emergentes, superando la simplicidad que caracterizaba a la modelización urbana tradicional (ALLEN, 1997).

El desarrollo de Sistemas de Información Geográfica (SIG), su integración con las modelizaciones urbanas preexistentes, las nuevas fuentes de datos y las innovaciones en las 
técnicas de análisis, han llevado a la tradición de modelos urbanos hacia un nuevo y renovado estadio en proceso de investigación y evaluación.

En los siguientes apartados se examinan los antecedentes teóricos, metodológicos y empíricos más destacados sobre la modelización urbana.

\section{LOS MODELOS DESDE LA ECOLOGÍA URBANA}

Este enfoque influido por el Darwinismo Social se basa en la creencia de que la conducta humana está determinada por los principios ecológicos, tales como la competencia impersonal, la selección, la invasión-sucesión, la dominación y la asimilación-segregación. Al igual que en la ecología vegetal, el grupo humano más poderoso podría obtener la posición más ventajosa en un entorno urbano determinado, por ejemplo, la mejor ubicación residencial. Este enfoque se remonta a la labor de la Escuela de Chicago de Ecología Humana de la década de 1920; los modelos más notables de este enfoque se presentan en la Figura 1: el modelo de anillos concéntricos de Burgess (1925), el modelo de los sectores de Hoyt (1939) y el modelo de núcleos múltiples de Harris y Ullman (1945).

El modelo de crecimiento urbano de Burgess (1925) se basó en la idea de que los diversos elementos de una sociedad urbana heterogénea y económicamente compleja promueven la competencia por los lugares favorables dentro de la ciudad. La competencia por el centro urbano provoca indefectiblemente una sucesiva expansión de los usos del suelo hacia la periferia de la ciudad, formando una serie de áreas concéntricas que rodean el centro.

Aunque el modelo de Burgess describe un patrón ideal de crecimiento urbano, no tuvo en cuenta diversos factores como las redes de transporte o la topografía, que pueden causar alteraciones en el patrón ideal. Sin embargo, su propuesta sirvió de base para la postulación de otros modelos de estructura y crecimiento urbano.

Así, posteriormente, Hoyt (1939) basándose en los estudios de los cambios de los patrones residenciales de 142 ciudades para los años 1910, 1915 y 1936, avanzó en un modelo de sectores en el que identificó áreas residenciales homogéneas que crecen desde el centro hacia la periferia en forma de cuña. En su modelo de sectores, este autor resalta la importancia de las vías de transporte para explicar el crecimiento urbano, como así también, considera los efectos que tienen las variaciones topográficas y los usos del suelo adyacente y cercano. Aunque limitado por la falta de exploración teórica, Hoyt (1939) sugiere que, desde el distrito central de negocios irradian diferentes sectores de viviendas más o menos deseables. Los

Sociedade e Território - Natal. Vol. 28, N. 2, p. 23 - 41. Jun./Dez. de 2016 
grupos de altos ingresos ocupan las áreas más codiciadas y, los demás, van situándose gradualmente en torno a las zonas privilegiadas. Las áreas residenciales de clase alta se extienden a lo largo de vías de transporte que garantizan buena accesibilidad al centro; como así también en terrenos altos o a lo largo de frentes de agua (si esas áreas no estaban ya ocupadas por las industrias manufactureras) y alrededor de las residencias de los líderes comunales. La consideración de los distintos factores en el modelado físico de uso del suelo urbano sugiere un problema para las pretensiones ecologistas de desarrollar un modelo general de uso del suelo, como así también heredan un fuerte anacronismo y simplismo en la explicación sobre la organización y distribución de los usos del suelo. Este modelo general ha sido posteriormente complejizado por Harris y Ullman (1945), alcanzando desde este enfoque ecológico una mejor aproximación a la comprensión de los procesos que estructuran el espacio urbano.

De acuerdo con los geógrafos Harris y Ullman (1945), los patrones de crecimiento urbano y los cambios de usos del suelo siguen los principios generales ecológicos identificados por Burgess (1925), como son: la formación de áreas especializadas de usos del suelo; la tendencia de ciertas actividades a estar situadas próximas entre sí y otras a repelerse sistemáticamente y el sometimiento de todas las actividades al proceso de selección espacial que el precio del suelo impone. Sin embargo, los autores argumentan que este crecimiento no se centra en un solo distrito central de negocios, sino en ciertos puntos de crecimiento o «núcleos», proponiendo así la «teoría de los núcleos múltiples». Esta teoría tiene en cuenta el hecho de que el espacio interno de las ciudades se debe tanto a las peculiaridades de sus respectivos emplazamientos como a la acción de fuerzas económicas y sociales de carácter más general. Asimismo, consideran a la historia de cada ciudad en particular como un factor importante en la configuración del desarrollo urbano.

Aunque los modelos clásicos de la ecología urbana establecen las primeras normas generales del crecimiento y estructuración del uso del suelo en una ciudad, estos modelos "han tendido a formular un marco teórico notablemente positivista, determinista, mecanicista y organicista" (GETTYS, 1940, p. 144). Por lo tanto, una fracción de ecologistas urbanos de la Universidad de California, van a perfeccionar la propuesta metodológica de la escuela sociológica de Chicago fundando lo que teóricamente se conoce como el «análisis de áreas sociales», marcando el comienzo de una «ecología social moderna». 
Esta propuesta tiene su origen en la tipología urbana de Shevky y Williams (1949) publicada en The Social Areas of Los Angeles, ampliada más tarde por Shevky y Bell (1955), quienes desarrollaron un esquema de clasificación concebido para categorizar deductivamente poblaciones de áreas censales en términos de tres factores básicos: rango social, urbanización y segregación. Este método considera la construcción de un índice combinado que clasifica cada área censal y finalmente las agrupa dentro de un área social definida.

La propuesta de estudiar las áreas sociales urbanas fue ganado un gran atractivo entre los investigadores de la década de 1960, quienes, con el aporte de la tecnología informática y los datos periódicamente disponibles, fueron gestando el «análisis factorial» o «ecología factorial» (SWEETSER, 1965; REES, 1971). Como señalan numerosos autores (ZARATE MARTÍN, 1991), lo que se propone la ecología factorial, no es un modelo de estructura urbana en sí, sino un instrumento matemático para el análisis sofisticado de la diferenciación del espacio urbano.

Sin duda alguna, más allá de su aporte claramente procedimental, las contribuciones más interesantes de la «ecología urbana moderna» al estudio de la estructura urbana, resultan de demostrar que los tres modelos clásicos, no se excluyen uno al otro sino que se sobreponen complementariamente (MURDIE, 1969). También, de posicionar la modelización urbana como un producto final de un razonamiento deductivo y sugerir la identificación de nuevas dimensiones de diferenciación para estudios específicos.

No obstante, el denso número de modelos empíricos sobre diversas ciudades en base a este enfoque fue encontrando demasiadas situaciones particulares y comportamientos excepcionales que terminaron por desvanecer los postulados ecológicos como teoría capaz de sustentar la modelización del crecimiento y desarrollo urbano. Sus críticas principales apuntan al empleo de sintéticos factores sociales y económicos como explicativos de las decisiones locacionales de los grupos sociales, dejando afuera factores importantes como aspectos subjetivos de los individuos que inciden en sus decisiones, interrelaciones complejas entre estructuras urbanas y procesos determinados acontecidos en otras escalas de análisis y las actuaciones de los planificadores y gobernantes que restringen las posibilidades personales de movilidad y localización en el espacio urbano. Así fue que el interés en este enfoque comenzó a perder protagonismo hasta desvanecerse a principios de 1970.

Sociedade e Território - Natal. Vol. 28, N. 2, p. 23 - 41. Jun./Dez. de 2016 


\section{LOS MODELOS DESDE LA FÍSICA SOCIAL}

Este enfoque constituye una vertiente metodológica de la «interacción espacial» propuesta por Ullman (1954), cuya orientación predominante es el diseño de modelos como analogías de las leyes físicas. Se consolidó a finales de 1950 dando origen a una incipiente «macrogeografía» (STEWARD y WARNS, 1958) de los fenómenos humanos que incluía, como categorías o dimensiones básicas, la población, la renta, la distancia y el tiempo. A partir de ello se pretendió, sobre todo, entender las regularidades y equilibrios generales en el tiempo y el espacio (WARNTZ, 1975), estudiando primero, comportamientos espaciales a escala regional y segundo, intensificar la especificidad hacia espacios locales (BUZAI y BAXENDALE, 2006).

Bajo esta perspectiva se utiliza la Ley de la gravitación de Newton como equivalente a la interacción social entre los lugares, proponiendo, a partir de ello, que el movimiento provocado por las actividades humanas, tales como los cambios de residencia o transporte a los lugares de trabajo, eran directamente proporcionales al producto de las masas (poblaciones $\mathrm{u}$ otras variables) de origen y destino, e inversamente proporcional al cuadrado de las distancias (también en términos de costo o tiempo) que las separan. Los modelos desarrollados a partir de esta analogía se conocen como «modelos de gravedad»o «modelos gravitatorios», y fueron aplicados inicialmente para la determinación de las áreas de influencia del comercio minorista (REILLY, 1929) y, posteriormente, a una amplia gama de fenómenos socioespaciales como estudios de migraciones, tráfico de pasajeros, transporte de mercancías e intercambio de información.

En muchos estudios gravitatorios en Geografía, la masa ha sido asimilada con el tamaño de la población, por ser un tema de interés y encontrarse disponible la información. Sin embargo, los datos de población suelen esconder importantes diferencias entre las regiones que afectarán, por ende, a las probabilidades de «interacción espacial». En este sentido, Isard (1960) sugería que de la misma manera que los pesos de moléculas de distintos elementos son desiguales, los pesos asignados a distintos grupos de personas deberían también variar, proponiendo la asignación de pesos diferente según las distintas zonas de Estados Unidos. Otra alternativa en este sentido podría ser "la multiplicación de la población de cada área por su renta per cápita media" (HAGGETT, 1994, p. 458). 
La física social fue ampliamente aplicada en los modelos de planificación urbana, y a partir de la evolución tecnológica que suponen los Sistemas de información Geográfica es posible una más fácil transferencia hacia un número mayor de usuarios e investigadores de procesos socioespaciales. No obstante, se les critica que no incorporan aspectos comportamentales (factores cualitativos) que motivan a los individuos a seleccionar un desplazamiento y trayecto particular en cada movilidad (trabajo, compras, ocio, residencial, etcétera). Además, son modelos agregados que hacen hincapié en comportamientos globales de individuos supuestamente de racionalidad uniforme. Asimismo, como los modelos se basan en supuestos análogos a las teorías de la física, las bases teóricas de interpretación, comprensión y explicación resultan insuficientes para analizar la complejidad que supone el espacio urbano.

\section{LOS MODELOS NEOCLÁSICOS BASADOS EN GRADIENTES}

El enfoque neoclásico se encuentra arraigado en la las teorías del equilibrio económico, siendo el modelo de von Thünen sobre la ubicación de la agricultura el primer modelo desarrollado bajo este enfoque, al que le sucedieron los modelos de Weber (1909), Lösch (1943) e Isard (1956).

Este enfoque fue construido sobre la creencia de que el proceso de desarrollo urbano es esencialmente un fenómeno económico, impulsado por mecanismos de mercado y por las fuerzas naturales de la competencia entre las actividades económicas y los grupos sociales, en un área urbana. Todos ellos se centran en los valores del suelo como factor básico para explicar la localización de los diferentes usos y cambios urbanos, e introducen tímidamente ciertas consideraciones de tipo social y cultural, en algunos casos.

Haig (1926) exponía prematuramente las ideas básicas sobre las que se basa este enfoque, centrando la atención en las relaciones entre localización, renta del suelo y costes de transporte, que dicho autor simplificará con la noción de «fricción del espacio». Así, los individuos, industrias, comercios y servicios compiten por el espacio en función de sus necesidades específicas de localización para obtener los máximos beneficios derivados de la disminución de la fricción y en función de las limitaciones de inversión (CARTER, 1972; ZARATE MARTÍN, 1991).

Sociedade e Território - Natal. Vol. 28, N. 2, p. 23 - 41. Jun./Dez. de 2016 
De este modo, un espacio urbano que se considera uniforme está habitado por hombres que dirigen sus acciones a la obtención del máximo beneficio. Según el modelo de «homo economicus» las actividades configuran una estructura urbana concéntrica (lugar de máxima accesibilidad global), siguiendo la disminución regular de los precios del suelo desde el centro hacia la periferia. Si bien los modelos neoclásicos se asemejan, en ciertos aspectos, a la teoría concéntrica y sectorial planteada por los representantes del enfoque ecológico (específicamente en relación al lugar asignado al distrito central de negocios y a la capacidad económica como factor central de análisis), éstos se interesaban más bien por las interrelaciones (y no por las descripciones) entre los distintos fenómenos económicos y sociales registrados entre las diferentes áreas urbanas. Además, se basan en el diseño de modelos de «gradientes» (en oposición a límites zonales), argumentando que existe un ritmo de cambio (no una ruptura marcada) de cualquier condición variable conforme se va produciendo un alejamiento respecto del centro de la ciudad hacia la periferia.

Estos modelos de estructura urbana se expresan gráficamente a través de curvas de renta que reflejan los precios que cada usuario del uso del suelo oferta por su localización en relación a su distancia al centro. Ejemplos pioneros destacados de estos modelos fueron desarrollados por Wingo (1961), Alonso (1964) y Lowry (1964), orientados al análisis de la organización interna del uso del suelo urbano.

Los modelos neoclásicos estándar fueron criticados por su simplificación, por considerarse irreal la suposición de un espacio isotrópico; la existencia de ciudades exclusivamente monocéntricas; la omisión de las influencias de los agentes productores del espacio urbano (entre los que se destacan los propietarios del suelo, las inmobiliarias y el poder regulador del estado) y de factores sociales, simbólicos y ambientales (CARTER, 1972). En momentos posteriores estos abordajes evolucionaron e incorporaron, entre otros elementos, múltiples centralidades, diferentes modos de transporte, factores externos, como la contaminación y los equipamientos colectivos; como así también, las variaciones de ingresos, las diferencias en las preferencias de los hogares, las variaciones en la calidad ambiental y la discriminación en los mercados de vivienda.

\section{LOS MODELOS DESDE LA PERCEPCIÓN Y EL COMPORTAMIENTO}

Este enfoque fue desarrollado a partir de la crítica de los conceptos excesivamente simplificados sobre la conducta humana implícitos en los planteamientos ecológicos y 
neoclásicos. A diferencia de estos enfoques que exploran sólo un aspecto de la conducta humana (la maximización del beneficio), el enfoque comportamental va a centrar la atención en las motivaciones de la conducta individual, sosteniendo que la manera en que los individuos perciben su entorno urbano determina, en gran parte, sus decisiones. La ciudad, es vivida por los ciudadanos que a través de las prácticas sociales y de la utilización cotidiana del espacio urbano van construyendo una imagen propia de la misma como fruto de sus percepciones particulares (CAPEL, 1975). Esta imagen, fruto de la información recibida individualmente por cada persona y también de sistemas de valores influidos por los medios socioculturales en el cual los individuos se desenvuelven y/o son manipulados por los medios de comunicación masivos, es una imagen diferenciada sustancialmente, tanto de la realidad misma, como de la visión del positivismo geográfico (BOSQUE MAUREL, 1986).

La vivencia individual o colectiva del espacio urbano puede, así, convertirse en un factor decisivo del comportamiento espacial de los ciudadanos, siendo fundamental su análisis para la comprensión de la estructura de usos del suelo urbano y la previsión racional del futuro de las ciudades en general y de cada caso en particular.

El trabajo de Lynch (1960) constituye el origen de cualquier estudio urbano desde la percepción y el comportamiento. El autor propone modelizar la estructura urbana a partir de cinco elementos básicos: 1) los nudos, centro de interacción de diferentes niveles que polarizan la práctica de sectores urbanos; 2) los barrios, sectores o distritos, en los que el habitante adquiere un cierto conocimiento y a los que se siente integrado o no; 3) los ejes, canales de observación e itinerario de desplazamiento que permitan acceder a los diversos equipamientos y nudos de la ciudad, a partir de los cuales se adhiere la familiaridad de los lugares muy diferenciada según la naturaleza y la velocidad del desplazamiento; 4) las barreras físicas y psicológicas, que limitan el marco de vida, su traspasamiento mediante de «puertas» más o menos anchas impone siempre un esfuerzo e incluso una renuncia a los criterios colectivos del grupo y; 5) los símbolos caracterizan y califican un espacio y organizan la localización, facilitando la familiaridad.

Estos elementos mensurables tienden a calificar de forma lógica los componentes físicos y funcionales del barrio para determinar a priori las posibilidades de su utilización y establecer comparaciones. Las elaboraciones de modelos en base a esta propuesta favorecieron, más allá de una descripción de las morfologías urbanas, los análisis psicológicos 
del espacio arquitectural, de las organizaciones sociales y de las relaciones entre un barrio y un medio urbano determinado.

Este enfoque no fue masivamente implementado para los estudios de estructuración urbana, debido a: su excesivo énfasis en el comportamiento individual y no de comportamientos generales; la delicada asociación entre la percepción y el comportamiento y la ardua tarea que implica la modelización de este procedimiento analítico. Sin embargo, ha existido una peramente evolución de los adherentes a este enfoque, especialmente en el campo de la difusión de las innovaciones y la movilidad residencial y, en quienes intentan estrechar los vínculos entre los modelos de comportamiento individual y restricciones sociales generales.

\section{LOS MODELOS DESDE EL ENFOQUE SISTÉMICO}

El modelado urbano en base en los conceptos de la Teoría General de Sistemas fue utilizado por primera vez en la década de 1960. Un sistema urbano se compone de un conjunto de elementos o subsistemas, tales como: población, suelo, empleo, servicios y transporte, por mencionar algunos. Todos los elementos dentro del sistema interactúan entre sí a través de mecanismos sociales, económicos y espaciales, mientras que también interactúan con los elementos naturales del entorno. El significado de cualquier elemento no depende de sí mismo, sino en sus relaciones con los demás. Estos vínculos existentes entre los diferentes elementos del sistema son los que determinan la evolución y posibles cambios en el mismo. Así, el acento en el enfoque de sistemas no está puesto en ningún elemento específico, sino en las conexiones y los procesos que enlazan todos los elementos (CHISHOLM, 1967).

A fin de representar la estructura y comportamientos de los sistemas, se han empleado una amplia gama de métodos matemáticos, incluyendo el análisis factorial, análisis de componentes principales, análisis multicriterio, la programación lineal y no-lineal, así como la dinámica de sistemas. Entre estos métodos, la técnica de «dinámica de sistemas» desarrollada por Forrester (1969) para simular inicialmente procesos industriales, tuvo una destacada adecuación a la dinámica urbana y procesos sociales en general.

Los primeros modelos de dinámica urbana creados por la escuela de Forrester no eran espaciales (FORRESTER, 1969), ya que la ciudad estaba constituida por una única zona, pero sí reflejaban la competencia entre diferentes usos del suelo dentro de un espacio urbano limitado. El suelo dedicado a vivienda y a actividades económicas, interactuando con el 
sistema demográfico permitía mostrar cómo actuaba la escala temporal en los procesos de crecimiento y deterioro urbano. Un concepto popularizado por estos modelizadores fue el de «comportamiento antiintuitivo» de los sistemas, tratando de mostrar a los decisores (funcionarios públicos) que las decisiones, con objetivos a corto plazo, podían tener efectos perversos en plazos más largos.

Esta técnica fue empleada como un laboratorio para la investigación estratégica y táctica en la toma de decisiones de gestión y planificación territorial urbana. Sin embargo, la falta de apoyo teórico y la imposibilidad de desagregación espacial de los resultados han limitado también su aplicación, tema que será complejizado posteriormente por una nueva corriente de modelización dinámica representada por las Escuelas de Leeds y de Bruselas.

El enfoque de sistemas representa una manera de construir modelos más allá de la simple relación de causa-efecto o de las relaciones estímulo-respuesta. Por lo tanto, estos modelos son ampliamente aceptados, especialmente por los planificadores de usos del suelo urbano que les permiten prever ciertas situaciones conflictivas asociadas al desarrollo urbano.

Sin embargo, este enfoque también presenta debilidades, ya que en algunos casos se utilizan fórmulas matemáticas extremadamente simplificadas para representar las complejas relaciones y flujos existentes entre los distintos elementos en la realidad. Así también, con el fin de lograr que ciertos modelos se vuelvan operativos, algunos terminan por abusar de las delimitaciones centrando la atención en pequeñas partes del sistema con excesiva independencia del conjunto y entorno general. En otros casos, se ha señalado una exacerbada rigidez al determinar la forma y magnitud de flujos e interconexiones a los fines de obtener sistemas controlados (LIU, 2009). Estas y otras limitaciones del enfoque basado en la Teoría General de Sistemas, han llevado a pensar que la complejidad de la realidad debe ser entendida y modelada por nuevas formas. En las últimas tres décadas, los estudios de procesos no-lineales, sistemas auto-organizados y teoría del caos han dado lugar a la aparición de una nueva modelización del espacio urbano entendido como un sistemas dinámico complejo.

\section{LOS MODELOS DESDE LOS SISTEMAS COMPLEJOS}

Si bien a partir de a la Teoría General de los Sistemas se logró modelizar con gran eficacia el comportamiento de gran cantidad de fenómenos, se mostraba también cierta 
insuficiencia ante circunstancias recurrentes en los estudios de procesos urbanos signados por la «incertidumbre» y la «imprecisión» que caracteriza a lo social.

A partir de la década de 1980, los desarrollos científicos sobre sistemas complejos nolineales, caóticos y auto-organizados han dado lugar a nuevas formas de interpretar y analizar las ciudades y su desarrollo, contribuyendo a considerables mejoras en la modelización del espacio urbano contemporáneo (BATTY y LONGLEY, 1994; ALLEN, 1997).

Estos estudios han permitido comprender el desarrollo urbano como un proceso irregular, resultante de las tensiones propias de un sistema abierto sujeto a decisiones internas y externas, donde tienen predominancia las configuraciones no-euclidianas y las conjunciones entre el espacio y el tiempo (BUZAI Y BAXENDALE, 2006).

El concepto de auto-organización resulta central e implica que la estructura urbana anterior incidirá en la forma urbana actual, y que ésta, a su vez, influirá en el patrón de uso del suelo urbano futuro. Estas ideas han dado lugar a la aparición de nuevas clases de modelos para simular el desarrollo urbano dentro de los que se destacan: la «agregación por difusión limitada» (DLA), la «simulación basada en autómatas» y los «modelos basados en agentes» (BENENSON y TORRENS, 2004; BATTY y LONGLEY, 1994; WHITE y ENGELEN, 1994; COUCLELIS, 1985).

La agregación por difusión limitada (DLA) es uno de los más importantes modelos de crecimiento basados en los principios de la geometría fractal, que ilustra la estructura irregular de un sistema que tiene el mismo grado de irregularidad en todas las escalas, característica denominada «autosimilitud». Este modelo, fue desarrollado por Witten y Sander (1981) para ilustrar una clase general de conducta que subyace a muchos fenómenos caracterizados por el crecimiento dendrítico, tales como, el crecimiento de escarcha en un cristal, iluminación, y chispas.

El estudio de diferentes configuraciones ha llevado a distinguir entre fractales perfectamente auto-similares (deterministicos) y fractales cuya autosimilitud es básicamente estadística (no-deterministicos o aleatorios); dentro de estos últimos se encuentra la agregación por difusión limitada (WRITTEN y SANDER, 1981).

Este procedimiento fue introducido por primera vez para modelar el crecimiento urbano por Batty (1991), quien siguiendo las normas propuestas por Witten y Sander (1981), desarrolló un modelo de DLA para modelar la dinámica del crecimiento urbano, que fue 
aplicado y probado en ciudades inglesas de tamaño medio como Taunton y Cardiff (BATTY y LONGLEY, 1994).

Por su parte, los modelos urbanos basados en la técnica de los «autómatas» también han surgido bajo el paradigma de un sistema de auto-organización, dentro de ellos, los más implementados son los «autómatas celulares» (AC). Los AC son herramientas que llaman poderosamente la atención para realizar estudio de sistemas auto-organizados debido a su simplicidad práctica y capacidad analítica de estructuras espacio-temporales complejas.

$\mathrm{Su}$ origen se remonta a los trabajos de John Von Neumann quién propone modelar máquinas que trabajando en forma autónoma (autómatas) tienen la posibilidad de autoreproducirse (BUZAI y BAXENDALE, 2006). Continuó con el aporte de StanislawUlam, quién creó a partir de los fenómenos de crecimiento de cristales, una red infinita desplegada como un tablero de ajedrez donde cada cuadrado de la red podría ser, esencialmente, una máquina que actuaría de acuerdo con un conjunto compartido de reglas. La configuración de la red cambiaría a medida que ocurrieran los pasos del tiempo discreto. Cada célula (casillero del tablero) contendría información que podría conocerse como su estado y, que consultaría la «tabla de reglas» para así determinar su estado en los siguientes momentos.

AAunque la técnica de los autómatas celulares se remonta a los orígenes de la computación digital, es a partir de la década de 1990 que esta técnica se ha utilizado para explorar el comportamiento de sistemas auto-organizados y modelar el proceso de crecimiento urbano, pudiendo citar los trabajos pioneros de White y Engelen (1994), White (1997) y una serie de estudios aplicados a diferentes ciudades tales como: Clarke, Hoppen y Gaydos (1997), Couclelis (1989) yCecchini (1996).

Otro tipo de modelización basada en autómatas son los «sistemas múlti-agente» (MAS), los cuales han demostrado excelentes resultados al aplicarlos en modelos urbanos (TORRENS y BENENSON, 2005). Los sistemas multi-agentes han sido diseñados como un conjunto de agentes autónomos que interactúan, cada uno con sus propias capacidades y objetivos, pero en conjunto se relacionan con un entorno común. Este tipo de modelo, opera bajo los mismos principios que el modelo de autómatas celulares, en donde cada agente se considera como un individuo autónomo (TORRENS, 2003), y gran parte del estado general del sistema, es explicado por comportamiento y características de los agentes. Sin embargo, existen varias distinciones entre los autómatas celulares y sistemas multi-agente. Una diferencia es que en el sistema multi-agente, la unidad básica de la actividad es el conjunto de 
agentes que representan a los individuos, planificadores o funcionarios del gobierno. Los agentes son autónomos, ya que son capaces de realizar acciones independientemente, sus actividades están dirigidas hacia el logro de tareas o metas definidas, y su influencia sobre el medio puede representarse en diferentes escalas.

Otra distinción entre los autómatas celulares y los sistemas multi-agente es que en los autómatas celulares, las células se fijan en la malla cuadriculada discreta, mientras que los agentes en los sistemas multi-agentes son entidades dinámicas y móviles que se pueden mover dentro de los espacios que "habitan" (TORRENS, 2003). Estos agentes también pueden procesar y transmitir información a sus vecinos mientras se mueven en el espacio. En consecuencia, las relaciones de vecindad entre agentes también son dinámicas: cuando los agentes individuales alteran su ubicación en el espacio, sus relaciones de vecindad también cambian. Esta técnica de modelado ofrece más flexibilidad debido a que sus agentes no sólo van a cumplir la función inherente de los autómatas (capacidad de cada agente de percibir su entorno), sino que, también, procesarán, evaluaran y actuarán en base a dichas percepciones conforme a ciertos principios de optimidad y consistencia, para satisfacer algún objetivo o finalidad, aproximándose así a ciertos comportamientos de la racionalidad o inteligencia humana (TORRENS, 2003). Por lo tanto, es claro que este tipo de enfoque de modelado es el que ofrece mayores potencialidades para el modelado urbano. No obstante, en la práctica, los modelos basados en agentes se encuentran menos difundidos en comparación con los modelos de autómatas celulares, y la mayoría de los modelos urbanos basados en agentes fueron formuladas en realidad como CA y reinterpretados como sistemas multi-agente (BENENSON y TORRENS, 2004; TORRENS, 2003).

\section{CONCLUSIONES}

La aplicación de modelos en las investigaciones científicas resulta importante, no sólo porque sirven como un medio para que las teorías se expresen en un lenguaje preciso, sino porque también permiten examinar críticamente las teorías e hipótesis integradas en ellos. Algunos autores señalan inclusive que el grado de desarrollo teórico en un campo del conocimiento es parcialmente equivalente a la medida en que se emplean modelos abstractos para su análisis y predicción.

Sociedade e Território - Natal. Vol. 28, N. 2, p. 23 - 41. Jun./Dez. de 2016 
Los modelos también son destacables en un sentido práctico, especialmente cuando se trata de estudiar los sistemas sociales, como lo es para los científicos y planificadores urbanos. A diferencia de los científicos de laboratorio, los investigadores sobre el espacio urbano rara vez pueden manipular los objetos bajo análisis para encontrar una mejor disposición o para descubrir sus propiedades naturales o leyes. Las escalas, costos y tiempo también obstaculizan las investigaciones, ya que suelen ser demasiado grandes para implementar análisis repetitivos, mientras que la experimentación controlada rara vez es una posibilidad al estudiar la sociedad. En este sentido, es que a través de la construcción de modelos, los investigadores encuentran una posibilidad de representar la estructura y funciones del sistema urbano como una alternativa para la comprensión y explicación (y en algunos aspectos hasta predicción) de este fenómeno en la realidad.

El campo del conocimiento que tiene mayores posibilidades de intentar una síntesis teórica respecto a los fenómenos que ocurren en las ciudades es la Geografía Urbana, ya que considera tanto los aspectos económicos, naturales y humanos sobre una base netamente espacial. Como marco metodológico la geografía urbana sirve tanto para estructurar los aspectos subjetivos y objetivos de la ciudad, como así también, contribuye a la solución de los problemas identificados dentro de su ámbito de acción.

Dentro de los aportes analizados, consideramos que el enfoque desde la Teoría de los Sistemas Complejos parece abarcar lo que los estudios urbanos han estado buscando durante mucho tiempo, la integración analítica entre espacio, tiempo y acción de la sociedad, para entender el comportamiento y evolución del patrón espacial que tradicionalmente denominamos estructura urbana.

\section{BIBLIOGRAFÍA}

ALLEN, P. M. Cities and regions as evolutionary, complex systems. Geographical Systems 4: 103-30, 1997.

ALONSO, W. Location and land use. Cambridge: Harvard University Press. 1964.

BATTY, M. Cellular automata and urban form: a primer. Journal of the American Planning Association. 63: 266-74. 1997.

BATTY, M. Generating urban forms from diffusive growth. Environment and Planning A. 23: 511-44. 1991.

BATTY, M. Urban models. Quantitative geography: a British view. (Wrigley N. y Bennett, R. J. Eds.). 181-91. London: Routledge and Kegan Paul. 1981.

Sociedade e Território - Natal. Vol. 28, N. 2, p. 23 - 41. Jun./Dez. de 2016 
BATTY, M. y LONGLEY, P. Fractal cities: a geometry of form and function. London: Academic Press. 1994.

BENENSON, I. y TORRENS, P. M. Geosimulation: automata-based modeling of urban phenomena. London: John Wiley. 2004.

BOSQUE MAUREL, J. El espacio urbano. Evolución y cambio en Geografía urbana. (GARCIA BALLESTEROS, Coord.). Teoría y práctica de la Geografía. Alhambra Universidad. 259-283. 1986.

BURGESS, E. W. The growth of city: an introduction to a research project. (PARK, R. E. BURGESS E. W. y MCKENZIE R. D. Eds.). The city. 47-62. Chicago: The University of Chicago Press. 1925.

BUZAI, G. y BAXENDALE, C. Análisis Socioespacial con Sistemas de Información Geográfica. Lugar editorial S.A. Bs. As. 2006.

CAPEL, H. La definición de lo urbano. Estudios Geográficos, 38-139: 265-301. Febreromayo 1975.

CARTER, H. El estudio de la geografía urbana. Colección Nuevo Urbanismo. Instituto de Estudios de Administración local. $2^{\circ}$ edición. Madrid. España. 1983.

CECCHINI, A. Urban modelling by means of cellular automata: generalised urban automata with the help on-line (AUGH) model. Environment and Planning B 23: 721- 32. 1996.

CHISHOLM, M. General systems theory and geography. Transactions of the Institute of British Geographers. 42: 45-52. 1967.

CLARKE, K. C., HOPPEN, S. y GAYDOS, L. J. A self-modifying cellular automaton model of historical urbanization in the San Franciso Bay area. Environment and Planning B 24: 247- 61. 1997.

COUCLELIS, H. Cellular worlds: a framework for modelling micro-macro dynamics. Environment and Planning A 17: 585- 96. 1985.

COUCLELIS, H. Macrostructure and microbehavior in a metropolitan area. Environment and Planning B 16: 141-54. 1989.

FORRESTER, J. W. Urban dynamics. Cambridge: The MIT Press. 1969.

GETTYS, W. Human Ecology and Social Theory. Social Forces. 18. 4. 1940.

HAGGETT, P. Geografía: una síntesis moderna. Barcelona. Omega. 1994.

HAIG, R. M. Toward an Understanding of the Metropolis: II. The Assignment of Activities to Areas in Urban Regions. The Quarterly Journal of Economics, 40 (3): 402-434. 1926.

HARRIS, C. D. y ULLMAN, E. L. The nature of cities. Annals of the American Academy of Political and Social Sciences 242: 7-17. 1945. 
HOYT, H. The structure and growth of residential neighborhoods in American cities. Washington, D.C.: U.S. Government Printing Office. 1989. 129-140.

ISARD, W. Methods of Regional Analysis an Introduction to regional science. MIT Press. 1960.

LIU, X. P.; LI X. y YEH, A. G. O. Multi-agent systems for simulating spatial decision behaviors and land use dynamics,Science in China (Series D). 49(11): 1184-1194. 2006.

LIU, Y. Modelling urban development whit Geographical Systems and Cellular Automata, CRC Press, New York. 2009.

LOWRY, I. S. A model of metropoles. Santa Monica, California: The Rand Corporation. 1964.

LYNCH, K. The image of the city. MIT Press. Cambridge. 1960. (Trad. Español: La imagen de la ciudad. Infinito, Buenos Aires, 1986).

MURDIE, R. A. Factorial Ecology in Metropolitan Toronto, 1951-1961: An Essay on the social Geography of the city. Research Paper 116. Department of Geography of Chicago. 1969.

REES, P. H. Factorial Ecology: an extended definition, survery, and critique of the field. Economic Geography. IV (2). 1971.

REILLY, W. Methods for the study of Retail Relationships (Research Monograph \# 4). University of Texas. Bureau of Bussiness Research. 1929.

SHEVKY, E. y BELL, W. Social area analysis. Stanford: California, Stanford University Press. 1955.

SHEVKY, E. y WILLIAMS, M. The social area of Los Angeles: analysis and typology. Berkeley: University of California Press. 1949.

STEWARD, J. Q. y WARNTZ, W. Macrogeography and social science. Geographical Review. 48: 167-184. 1958.

SWEETSER, F. L. Factorial Ecologý: Helsinki 1960. Demography. 2: 372-386. 1965.

TORRENS, P. M. Automata-based models of urban systems. (LONGLEY, P. y BATTY, M. Eds.) Advanced Spatial Analysis: the CASA Book of GIS. 61- 81. Redlands, California: ESRI Press. 2003.

TORRENS, P. M. y BENENSON, I. Geographic Automata Systems. International Journal of Geographical Information Science. 19: 385-412. 2005.

ULLMAN, E. L. Geography as spatial interaction. (Boyce, R. R. ed.). University of Washington Press. Seattle. 13-27 (versión ampliada y reeditada). 1980 (1954).

WARNTZ, W. La nueva Geografía como teoría de sistemas espaciales ¿Cuenta mucho la vieja física social? (CHORLEY, R. J.). Nuevas tendencias en Geografía. Col. N. U. 15. IEAL. Madrid. 1975.

Sociedade e Território - Natal. Vol. 28, N. 2, p. 23 - 41. Jun./Dez. de 2016 
WHITE, R. Cities and Cellular Automata. Discrete Dynamics in Nature and Society, Vol. 2: 111-125. 1997.

WHITE, R. y ENGELEN, G. Cellular dynamics and GIS: modelling spatial complexity. Geographical Systems 1: 237- 53. 1994.

WINGO, L. Transportation and urban land. Baltimore, MD: John Hopkins University Press. 1961.

WITTEN, T. A. y SANDER, L. M. Diffusion-limited aggregation: a kinetic critical phenomenon. Physical Review Letters. 47: 1400-03. 1981.

ZARATE MARTÍN, A. El espacio interior de la ciudad. Espacios y Sociedades, serie general N 12 . Editorial Síntesis. Madrid. 1991.

Recebido em Abril de 2016

Aprovado em Agosto de 2016

Publicado em Dezembro de 2016

Sociedade e Território - Natal. Vol. 28, N. 2, p. 23 - 41. Jun./Dez. de 2016 